\title{
Enseñar comprensión de textos a escolares con trastornos del espectro autista: Secretos desde la experiencia
}

\section{Teaching Reading Comprehension to School Children With Autism Spectrum Disorders: Secrets From Experience}

\section{Ensinar a compreensão de textos para crianças em idade escolar com Distúrbios do Espectro do Autismo: segredos da experiência}

Universidad de Pinar del Río Hermanos Saíz Montes de Oca

Pinar del Río, Cuba

giselvis.aguiar@upr.edu.cu

https://orcid.org/0000-0001-5265-6093

Débora Mainegra-Fernández Universidad de Pinar del Río Hermanos Saíz Montes de Oca

Pinar del Río, Cuba dmainegrafernanadez@gmail.com https://orcid.org/0000-0003-0811-0629

Olivia García-Reyes

Universidad de Pinar del Río Hermanos Saíz Montes de Oca

Pinar delRío, Cuba olivia.garcia@upr.edu.cu https://orcid.org/0000-0003-0318-0628

Recibido • Received • Recebido: 21 / 11 / 2018

Corregido • Revised • Revisado: 27 / 01 / 2020

Aceptado • Accepted • Aprovado: 01 / 04 / 2020

Resumen: En el presente ensayo se reflexiona acerca de las vías que pueden propiciar el aprendizaje de la comprensión textual en escolares con trastornos del espectro de autismo (TEA), a partir de las experiencias de trabajo de las autoras. Se ofrecen un grupo de ideas que pueden contribuir a mejorar la labor del personal docente que atiende estudiantes con necesidades educativas especiales de este tipo, a partir de ejemplos que facilitan su empleo. Entre las vías novedosas que se ofrecen están el emparejamiento y la recodificación desde un patrón visual y las rutinas estructuradas basadas en la utilización de apoyos visuales, a partir de la potencialidad que representa la memoria visual de estos niños y niñas.

Palabras claves: Educación; comprensión de textos; necesidades educativas especiales; autismo. 
http://doi.org/10.15359/ree.24-2.22

ROR: https://ror.org/01t466c14 Universidad Nacional, Costa Rica

http://www.una.ac.cr/educare

educare@una.cr

\begin{abstract}
The essay reflects on the ways children with Autism Spectrum Disorders (ASD) can learn reading comprehension at school, based on the work experiences of the authors. It offers a set of ideas, from examples facilitating their use, that can help improve the work of teachers in charge of students with special educational needs due to ASD. Some novel ways offered are pairing and recoding from a visual pattern and structured routines based on the use of visual supports, based on the potential of the visual memory of these children.
\end{abstract}

Keywords: Education; reading comprehension; special educational needs; autism.

Resumo: Neste ensaio, refletimos sobre as formas que ajudam na aprendizagem da compreensão textual para estudantes com Transtornos do Espectro do Autismo, com base nas experiências de trabalho das autoras. São propostas um grupo de ideias que podem ajudar a melhorar o trabalho de professores que atendem estudantes com necessidades educacionais especiais desse tipo, a partir de exemplos que facilitam seu uso. Entre as novas formas oferecidas estão o emparelhamento e a recodificação de um padrão visual e rotinas estruturadas baseadas no uso de figuras visuais, com base no potencial representado pela memória visual dessas crianças.

Palavras chaves: Educação, compreensão de textos; necessidades educacionais especiais; autismo.

\title{
Introducción
}

La comprensión de textos, como proceso, activa interacciones diversas entre personas autoras, lectoras, texto y contexto desde una perspectiva sociocultural transversalizada por el universo del saber de cada uno de los componentes personales. En la asignatura Lengua Española constituye un reto pedagógico para la educación de las personas con necesidades educativas especiales, de manera particular para escolares con trastornos del espectro de autismo (en adelante TEA), ya que dadas las carencias que presentan en el desarrollo de la comunicación, requieren de un trabajo correctivo-compensatorio que les permita interactuar socialmente de forma adecuada.

La asignatura Lengua Española ocupa un lugar determinante por su contribución al desarrollo de habilidades comunicativas mediante la práctica sistemática de la lengua. Teniendo en cuenta las limitaciones en el lenguaje receptivo de escolares con TEA, el desarrollo de la comprensión textual es de vital importancia para su aprendizaje escolar y su inclusión social.

Por otra parte, dentro de las macrohabilidades del aprendizaje de lenguas se incluyen las de escuchar, hablar, leer y escribir, y como habilidades transversales que implican el logro de estas, se conciben las de comprender y construir textos. Teniendo en cuenta que escolares con TEA presentan necesidades en la comunicación social, el logro de la comprensión textual resulta de vital importancia en esta educación, de modo que esta le sirva de referencia para la posterior construcción de textos, sobre la base de modelos lingüísticos; sin embargo, no siempre se realiza la labor que ello requiere. 
Las posibilidades de estos grupos de escolares para la inclusión son un desafío para los cuerpos educativos, toda vez que los procesos de aprendizaje presentan diferencias significativas lo cual está dado, entre otras razones, por la variabilidad en los niveles de desarrollo y la diversidad en el contexto áulico.

El Inventario de las dimensiones del espectro autista (IDEA), creado por Rivière (1997), instrumento fundamental utilizado por psiquiatras y docentes en los procesos de diagnóstico y evaluación psicopedagógica, solo evalúa áreas de desarrollo, por su carácter eminentemente clínico, por lo que en la práctica educativa no se valora y diseña adecuadamente el proceso de comprensión textual. Todo ello nos motivó a realizar el estudio que les presentamos en este ensayo, cuyo objetivo es compartir vías para lograr la comprensión textual en escolares con trastornos del espectro de autismo en las clases de Lengua Española.

La propuesta tiene sus fundamentos generales en ciencias como la filosofía, sociología, psicología, biología, lingüística, pedagogía, didáctica general y particular de la lengua española, los cuales permiten el sustento teórico y metodológico de la concepción didáctica que se propone.

La filosofía materialista dialéctica en general "...brinda la plataforma filosófica que sirve de faro para orientar su rumbo hacia el tipo de hombre que se requiere preparar, para asumir con éxito los retos de la realidad que impone la vida actual y futura"(Chávez Rodríguez, 2011, p. 1) y la "Filosofía de la Educación, [en particular], ofrece la brújula orientadora, la guía teórica necesaria para no perder el rumbo en el misterioso [proceso] de enseñar y aprender" (Chávez, 1995, citado por Paz Aguilera, 2011, Desarrollo, párr. 20).

La filosofía de la educación permite comprender el origen del proceso de enseñanzaaprendizaje de escolares con TEA, ayuda a interpretar las metas y objetivos de la asignatura Lengua Española para el desarrollo de la comprensión textual y permite expresar, de manera coherente, las necesidades y posibilidades educativas de la sociedad y del individuo, reconoce la educabilidad de las personas con TEA y el porqué y el para qué se educan.

El carácter social de esta concepción descansa sobre la base del enfoque no clasista de la educación en Cuba, específicamente de la educación especial y en la concepción del ser humano como ser social, en su unidad bio-psico-social. En esta juega un papel primordial la comunicación social de escolares con TEA, como proceso de su desarrollo individual, lo que contribuye, de manera efectiva, al aprendizaje. En este, las vivencias y los otros seres aportan recursos y apoyos favorecedores de la comprensión de diferentes tipos de textos, con una visión positiva en sus múltiples relaciones macrosociales (escuela, familia, comunidad) desde el proceso de comprensión textual contextualizado y significativo, en su contribución a la inclusión social. 
http://doi.org/10.15359/ree.24-2.22

ROR: https://ror.org/01t466c14 Universidad Nacional, Costa Rica

http://www.una.ac.cr/educare

educare@una.cr

Los fundamentos psicológicos se asumen de los postulados del enfoque históricocultural de Vigotski (1987), pues su esencia humanista aporta definiciones importantes para la comprensión y potenciación del desarrollo de escolares con TEA, entre ellos: la enseñanza como guía y conductora del desarrollo, la relación entre lo biológico y lo social, la ley genética fundamental del desarrollo, la ley de la mediación de los proceso psíquicos, el rol de las vivencias en el desarrollo psíquico, la situación social del desarrollo, la zona de desarrollo próximo (ZDP) y la unidad de lo afectivo y lo cognitivo en el proceso de enseñanza- aprendizaje.

Es importante conocer lo que escolares con TEA hacen por sí solos o solas y en lo que necesita la cooperación de otra persona, para poder determinar los objetivos a lograr, la secuencia de contenidos que requiere para alcanzar los objetivos propuestos y los medios que se necesitan para asimilar los contenidos, así como los niveles de ayuda que deben ofrecerse para contribuir al logro de los objetivos en correspondencia con las orientaciones metodológicas.

Las acciones y los medios se seleccionan a partir de las cualidades de los períodos sensitivos para el desarrollo de las funciones psíquicas superiores, la situación social del desarrollo, así como las potencialidades y necesidades de la niñez con TEA. La utilización de diferentes formas de abordarlo toma en cuenta los mecanismos de corrección y compensación para prevenir las alteraciones secundarias que pueden manifestarse y agravar su estado en su comunicación social.

Desde lo biológico, la concepción se sustenta en que las personas con TEA, al igual que los demás seres humanos, constituyen una unidad biopsicosocial, en cuyo desarrollo se conjugan factores biológicos y sociales (Vigotsky, 1987). La comprensión de los factores biológicos en el desarrollo como proceso general y en particular de la psiquis humana toma en cuenta la dinámica de la relación estructura-función, causa-efecto, unidad-diversidad y esencia-fenómeno.

La interacción "...organismo humano ... [y] ambiente como fundamento de la relación dialéctica que se establece entre lo biológico, lo psicológico, lo social" (Hernández Pérez et ál., 2010, Orientaciones metodológicas, párr. 3)“y el papel rector del sistema nervioso en su integridad funcional" (Hernández Pérez et ál., 2010, Orientaciones metodológicas, párr. 7) permite, no solo la fundamentación científica de las alteraciones del desarrollo que conducen al autismo, sino también la comprensión de las posibilidades del trabajo correctivo-compensatorio.

Si bien los trastornos del espectro de autismo tienen como base una alteración en el sistema nervioso central (SNC), donde se pueden presentar la combinación de factores genéticos o ambientales, que se expresan en las peculiaridades del desarrollo, especialmente alteraciones variadas en la comunicación social y comportamiento, intereses o actividades restringidas y repetitivas; la multiplicidad de factores que se conjugan en su origen y evolución; la variabilidad de las manifestaciones y la heterogeneidad del trastorno; todo esto, debidamente tratado a partir de una adecuada organización de las condiciones sociales y educativas en las que se desenvuelve el sujeto, constituye el pilar de los procesos compensatorios y, con ello, de su trasformación. 
Desde el punto de vista lingüístico, la concepción parte del valor que tiene el logro de la comunicación social para escolares con TEA y su interacción en los diferentes contextos en que se desarrollan, a través de las diferentes formas de comunicación tanto verbal como no verbal y asumiendo los roles de sujetos emisores y receptores con todo lo que ello implica.

A partir de la lingüística del texto (van Dijk (2000), se pueden establecer las relaciones entre diferentes aspectos que, sin duda, favorecerán el desarrollo de la comunicación social, como son: comprender diferentes textos a partir de su audición o lectura, construir los textos que necesite mediante la adecuada selección léxica para interactuar en su entorno, emplear correctos modos de actuación al asumir diferentes actitudes ante determinadas situaciones, utilizar tanto la expresión verbal como la no verbal en su comunicación, recurriendo para ello a las formas elocutivas.

La concepción, además, se fundamenta en la pedagogía general y especial de Labarrere Reyes y Valdivia Pairol (1988) y los postulados de Guerra et ál. (2014) a partir de la relación entre los componentes del proceso de enseñanza aprendizaje. Por ello, los objetivos, contenidos, métodos, medios, formas de organización y evaluación están conscientemente organizados y orientados a un fin: el desarrollo de la comprensión textual en escolares del primer ciclo con TEA, que contribuirá al proceso de inclusión social, a partir de la consideración hipotética de que la atención educativa que se estructura sobre esa base, conduce y guía el desarrollo.

Las principales ventajas de esta propuesta que les presentamos, a juicio de las autoras, están en su carácter humanista, optimista, colaborativa grupal, personalizada, transformadora y dinámica.

Se parte de que cada escolar, con sus potencialidades y necesidades, es el centro del proceso docente educativo, desde una concepción humanista, donde el maestro o maestra asume el rol principal de mediación de las relaciones que establece cada estudiante con el grupo. Se pretende desarrollar la comprensión de diferentes tipos de textos, de modo que les permita una mejor comprensión del medio social, lo que repercutirá en el desarrollo de la personalidad.

Revela una concepción didáctico- metodológica porque ofrece, al personal docente, argumentos científicos para dirigir el proceso de comprensión textual en escolares con TEA, acorde a las condiciones históricas y sociales existentes. Al mismo tiempo, les hace conscientes desde sus objetivos, contenidos, métodos y orientaciones metodológicas de las potencialidades que tienen para incidir en la formación de estos grupos escolares de acuerdo con las exigencias de la sociedad en la cual viven.

Esta concepción didáctica es colaborativa grupal porque en ella se vincula al personal docente de las diferentes áreas que, como mediador, ofrece diversas ayudas pedagógicas y tiene la responsabilidad de orientar a la familia, agente potenciador importante de la estimulación del desarrollo de sus hijos e hijas. 
Permite, desde un enfoque personalizado, atender, de forma individualizada, a cada escolar, porque propone tareas de aprendizaje variadas en correspondencia con los niveles de desarrollo de cada cual.

Es transformadora porque tiene como fin el desarrollo de la comprensión textual de escolares del primer ciclo con TEA, que les permita la asimilación de conocimientos, habilidades y normas sociales, en función de favorecer una mejor inclusión social.

La concepción didáctica que se presenta asume el concepto de texto de van Dijk (2000) y los postulados del enfoque cognitivo, comunicativo y sociocultural de Roméu Escobar (2006), sus fundamentos teóricos y su concepción del lenguaje como medio esencial de cognición y comunicación humana y de desarrollo personal y sociocultural del individuo. Con ello se favorece el aprendizaje activo del idioma; el desarrollo de la comunicación escrita y del pensamiento; se posibilita establecer relaciones interdisciplinarias, y preparar a estos grupos de escolares para situaciones sociales de comunicación.

\section{Algunas ideas para enseñar comprensión textual a escolares con TEA}

Las ideas que se presentan a continuación no se apartan del contenido de lectura, escritura, redacción y comprensión de la Lengua Española que aparece en los programas de la asignatura en el primer ciclo. Son una síntesis sobre el deber ser de la concepción del proceso que las autoras proponen y constituyen una guía orientadora para perfeccionar el proceso de comprensión textual en escolares del primer ciclo con TEA, desde la asignatura Lengua Española. A continuación se presentan y explican:

Primera idea. El proceso de comprensión textual en escolares del primer ciclo con TEA ha de ser planificado a partir de un sistema de clases flexibles (puede haber más de una clase dedicada a un nivel de comprensión) que lo orienten, organicen y lo conduzcan desde la singularidad de los recursos didácticos y la contextualización de tareas de aprendizaje a la variabilidad del TEA.

\section{Argumentos}

Es la idea que permite al personal docente la comprensión de la concepción didáctica que se propone.

Las dificultades en el lenguaje receptivo en este tipo de escolar se manifiestan en el proceso de comprensión textual, evidenciadas en los problemas para el procesamiento consciente y autorregulado al abordar las tareas de comprensión e incide directamente en el tránsito por los niveles de comprensión textual. Esta necesidad requiere que, en el proceso de comprensión textual, desde la asignatura Lengua Española el maestro o maestra tenga en cuenta que escolares del primer ciclo con TEA necesitan: 
- El aprendizaje de rutinas estructuradas para abordar las tareas de comprensión al enfrentarse a un texto determinado, por lo que necesitan mayor tiempo en el tránsito por los niveles de comprensión.

- Recursos y apoyos visuales como: agenda pictográfica, objetos reales, visionado de fotos, pictografías, videos; que posibiliten que manipule, descubra las propiedades externas, que enriquezca sus estructuras cognitivas, y estas puedan ser utilizadas para aprender el significado de las palabras y facilitar su posterior comprensión en sintagmas y oraciones.

- Tareas de aprendizaje diferenciadas que consideren la variabilidad en su desarrollo.

Para ello es necesario que la maestra o maestro tenga en cuenta tres aspectos fundamentales, que se expondrán a continuación en forma sintetizada.

1. Planificar el sistema de clases de la asignatura Lengua Española, a partir de la aplicación del currículo básico de la Educación Primaria, con adecuaciones a los componentes didácticos, donde el trabajo metodológico con la lectura y la comprensión de textos debe caracterizarse por la flexibilidad, para lo cual se hace necesario utilizar una misma lectura en un sistema de clases de cinco días para lograr que escolares con TEA transiten por los niveles de comprensión textual (traducción, interpretación y extrapolación), dosificadas de la siguiente manera:

Clase 1. Traducción del texto.

Clase 2 y 3 . Interpretación del texto.

Clase 4 y 5. Extrapolación del texto.

Este sistema de clases, además, deberá tener en cuenta la variabilidad del desarrollo y la heterogeneidad del TEA, por lo que se deberán adecuar las categorías didácticas de manera que el personal docente estimule en este grupo de escolares la posibilidad de aprender a ser y aprender a convivir, en el marco de las relaciones sociales que establezca, aspecto que se ampliará en la contextualización de los criterios didácticos de esta concepción.

2. Utilizar recursos didácticos, considerándolos mediadores de la actuación docente, elementos de apoyo, que pueden ser empleados y enriquecen el proceso de comprensión textual en escolares del primer ciclo, cualificándolo de manera singular en escolares con TEA, quienes responden, en primer lugar, a las características individuales y a la variabilidad del desarrollo. Como recursos didácticos se valoran altamente: el lenguaje facilitado, el aprendizaje cooperativo y la dramatización.

3. Planificar tareas de aprendizaje contextualizadas a la variabilidad en el desarrollo de escolares con TEA. 
http://doi.org/10.15359/ree.24-2.22

ROR: https://ror.org/01t466c14 Universidad Nacional, Costa Rica

http://www.una.ac.cr/educare

educare@una.cr

En el proceso de preparación del sistema de clases, el personal docente deberá realizar una valoración profunda sobre en qué medida el contenido de enseñanza que va a estructurar aporta a la instrucción, educación y desarrollo de escolares con TEA. Deberá cuidar que en la planificación de sus clases se cumplan los fundamentos y principios didácticos de la concepción que se propone, así como la presencia de las categorías del proceso de enseñanza-aprendizaje desarrollador.

Se considera necesario que el personal docente tenga en cuenta una serie de elementos, los cuales constituyen aspectos organizativos fundamentales de partida para la planificación del sistema de clases, los cuales se exponen a continuación:

- Conocer el diagnóstico del escolar o de la escolar.

- Conocer detalladamente las características del desarrollo a partir del proceso de evaluación psicopedagógica y el diseño de su estrategia educativa, en la que determine potencialidades y necesidades en elárea de la comunicación social; es importante conocer las necesidades en el lenguaje receptivo para poder desplegar todas las posibilidades instructivas y educativas que potencien el desarrollo de la comprensión textual.

- Dominar los objetivos y contenidos esenciales de cada una de las unidades didácticas del programa de la asignatura en el ciclo y en el grado (Análisis metodológico).

- Adaptar las exigencias de los objetivos, contenidos o el ajuste de las tareas, teniendo en cuenta la variabilidad en el desarrollo de escolares con TEA. La adaptación no debe provocar supresión o alejamiento de los objetivos, contenidos o tarea en cuestión, porque las intenciones educativas para estos grupos escolares son iguales a las del resto de escolares del primer ciclo de la Educación Primaria.

- Considerar la contextualización de los objetivos, métodos, medios de enseñanza, las formas de organización, la evaluación para el desarrollo de la comprensión textual teniendo en cuenta la variabilidad del desarrollo de escolares del primer ciclo con TEA.

- Considerar las potencialidades educativas del contenido para vincularlo con los diferentes contextos donde se desarrolla cada escolar.

Los elementos anteriores permitirán al maestro o maestra la comprensión exacta del volumen de información que han de aprender sus escolares (exigencias a alcanzar), así como prever con anticipación cómo se han de trabajar los niveles de comprensión textual en el transcurso del sistema de clases.

Segunda idea: En el proceso de comprensión textual en escolares con TEA, el texto ha de ser decodificado teniendo en cuenta las particularidades en el emparejamiento y la recodificación desde un patrón visual, como métodos didácticos fundamentales. 


\section{Argumentos}

Se propone realizar la traducción o decodificación del texto en la primera clase del sistema planificado por el personal docente. El objetivo principal en esta clase será decodificar un código, en este caso el de la letra impresa para que esta tenga un significado. Podrá realizarlo mediante dos formas fundamentales de decodificación que se particularizan al tener en cuenta la variabilidad del desarrollo de escolares del primer ciclo con TEA.

- Emparejamiento: emparejar la palabra impresa con un patrón conocido (visual), a partir de la relación directa y explícita entre la imagen del objeto y la palabra escrita, de esta forma se activa el significado de la palabra en la memoria de cada escolar. Así, el sujeto lector va adquiriendo, de forma paulatina, un vocabulario visual. En el vocabulario visual subyace toda una estructura de conocimiento previo en escolares con TEA.

- Recodificación: si no conoce la palabra impresa, pregunta a otra persona más competente, la pronuncia, busca en el diccionario según las habilidades del grado para su uso elaborando el significado en la mente del sujeto lector. Es de suma importancia, además, trabajar con los verbos de las acciones mentales por las dificultades en teoría de la mente que presentan los escolares con TEA y proporcionar medios de enseñanza (como láminas, vídeos, software, calendarios o agendas pictográficas, tarjetero, etc.) que les ayuden a centrar la atención en las más importantes palabras o frases del texto para su comprensión, así desarrollan habilidades que constituyen los primeros pasos para la aplicación de la técnica del lenguaje facilitado.

Los ambientes bien estructurados apoyan las conductas apropiadas y ayudan a la comprensión, por lo que se recomienda la utilización de procedimientos como el uso de fotografías, pictogramas, símbolos para la comprensión de los mensajes que se quieran trasmitir. Se deben disponer de situaciones de aprendizaje, secuencias de acciones, historias sociales, láminas, agenda pictográfica de verbos mentales u acciones, panel de intenciones, entre otros, de modo que contribuyan a la comprensión textual.

Se deben estructurar las actividades necesarias para el proceso de comprensión y proporcionar apoyos que faciliten su realización autónoma. Requiere que la maestra o maestro, en conjunto con el sujeto escolar:

- Defina cada uno de los procedimientos para la realización de la actividad (leo, releo, identifico el tipo de texto, busco las palabras que no conozco, busco el significado por diferentes vías, busco los verbos o las acciones que realizan los personajes y las comprendo, selecciono la idea central del texto).

- Disponga [los medios de enseñanza necesarios] pararealizar la tarea de manera que ayude a los [escolares y las escolares] a hacerla autónomamente, tanto por la forma con la que se presenta el material, como por la presencia de etiquetas, colores y otro tipo de señales que les indiquen lo que deben hacer. (Escribano Burgos et ál., 2010, p.162) 
Tercera idea: El proceso de comprensión textual en escolares conTEA ha de desarrollarse a través de rutinas estructuradas basadas en la utilización de apoyos visuales como procedimientos didácticos orientadores para el logro de la interpretación.

\section{Argumentos}

Se propone realizar la interpretación del texto en la segunda y tercera clase del sistema planificado por el maestro o maestra. El objetivo principal en estas clases será interpretar el texto, en las cuales escolares con TEA deberán comprenderlo en el nivel literal e inferencial.

Para la comprensión literal, escolares deberán establecer relaciones sencillas entre las ideas principales, ordenar secuencias, realizar pequeñas comparaciones, establecer las relaciones causa-efecto. (Diciendo de qué o quién se habla en el texto, qué se dice, qué ocurrió, cómo o por qué ocurrió). Requiere en esencia de la habilidad de:

Relacionar: establecer conexión, correspondencia entre varios elementos, ya sea de causa y efecto, de comparación, ordenamiento, asociación, expresada a través de un lenguaje (oral o con apoyo de los sistemas alternativos de la comunicación, preguntas sencillas con respuesta, preguntas de selección múltiple, lámina, dibujo, dramatización).

La maestra o maestro deberá hacer uso del lenguaje oral con claridad, realizar preguntas sobre el texto de forma clara y sencilla, podrá realizar breves comentarios sobre las acciones de los personajes u otra cuestión particular del texto, brinda confianza y seguridad.

Especial atención brindará "[al] apoyo emocional mediante el uso de gestos, la mirada, la voz y otras expresiones emocionales que muestran disponibilidad y cercanía afectiva" (Escribano Burgos et ál., 2010, p.163), así como al papel de las vivencias para la comprensión del texto.

La utilización de preguntas de selección múltiple y enlace de oraciones relacionadas entre sí constituyen procedimientos fundamentales a emplear por el personal docente, teniendo en cuenta las potencialidades de escolares con TEA de asimilar los aprendizajes visuales, gracias a su excelente memoria visual y memoria mecánica asociativa.

Se recomienda el uso de medios de enseñanza visuales como: agenda de la asignatura donde se expongan las acciones o tareas de comprensión, agenda de verbos $u$ acciones, diccionario pictográfico con las palabras del vocabulario de la asignatura en el grado, panel de peticiones, tarjetero pictográfico, etc.

En el desarrollo de la tercera clase del sistema propuesto, se deberá lograr la comprensión inferencial que requiere de un análisis más amplio de las ideas que se están leyendo, en los que el sujeto receptor (escolar) asume una posición ante el texto, opina, aprovecha su contenido y "Io aplica en otros contextos ... reacciona ante lo leído y modifica su conducta, trata de resolver problemas" (Manzano Díaz, 2007, p. 163), siempre teniendo en cuenta la variabilidad en el desarrollo del escolar con TEA.

Giselvis Aguiar-Aguiar, Débora Mainegra-Fernández y Olivia García-Reyes

Los artículos de la Revista Electrónica Educare del Centro de Investigación y Docencia en Educación de la Universidad Nacional, Costa Rica, se comparten bajo términos de la Licencia Creative Commons: Reconocimiento, № Comercial, Sin Obra Derivada 3.0 Costa Rica. Las autorizaciones adicionales a las aquí delimitadas se pueden obtener en el correo: educare@una.cr 
Requiere de las habilidades:

- Deducir: El logro de esta habilidad es esencial en escolares con TEA a partir de textos que presenten situaciones cotidianas como propagandas, avisos, recetas; donde a partir de una idea general contenida en el texto, se puedan elaborar otras relacionadas con el tema en cuestión, para lo cual el aprendizaje cooperativo es necesario para que estudiantes con TEA utilicen a los "otras personas" en la construcción de su propio aprendizaje.

- Ejemplificar: Demostrar o ilustrar con ejemplos lo que se dice. Se sugiere la utilización de la dramatización como recuso didáctico.

Las rutinas estructuradas basadas en la utilización de apoyos visuales serán el procedimiento fundamental a tener en cuenta para garantizar el éxito de la interpretación del texto. Se deberán utilizar las representaciones gráficas o pictografías que muestran las distintas acciones o pasos que componen una actividad, para que escolares con TEA comprendan las acciones que la integran y puedan utilizarlas como guía para realizar las tareas de comprensión.

Es muy importante utilizar la demostración con estudiantes en el aula, para ayudar a escolares con TEA a partir de la imitación, a resolver las tareas de comprensión de un texto o de una situación en la vida social, definiendo las metas y los pasos que se realizarán para alcanzarlas y, posteriormente, para valorar su ejecución utilizando diferentes recursos didácticos (el aprendizaje cooperativo, para potenciar la colaboración y ayuda mutua, el intercambio y la socialización de ideas entre escolares y el lenguaje facilitado para darles herramientas que les permitan comprender el texto en cualquier contexto).

Cuarta idea: El proceso de comprensión textual en escolares del primer ciclo con TEA ha de basarse en la utilización de una agenda pictográfica, como medio didáctico para el logro de la extrapolación en los contextos sociales.

\section{Argumentos}

Se propone realizar la extrapolación del texto en la cuarta y quinta clase del sistema planificado por el maestro o maestra. El objetivo principal en estas clases será extrapolar el texto.

En el desarrollo de estas clases el personal docente estimulará a sus estudiantes para que logren aplicar lo comprendido, transfiriendo los conocimientos a situaciones de la vida cotidiana, por lo que es fundamental que en estas clases los contenidos se relacionen y organicen en contextos sociales, como por ejemplo: centros comerciales y de servicios, parques, etc. Los métodos fundamentales son: los orales, visuales y prácticos. 
El medio de enseñanza fundamental en estas clases es la agenda pictográfica para representar con pictogramas la secuencia de acciones a realizar para comprender un texto en un contexto social determinado; podrán ser utilizados otros como: los pictogramas, fotografías, agenda pictográfica de verbos mentales u acciones, panel de intenciones, tarjetero pictográfico, que permitan a escolares con TEA organizar y seguir las rutinas estructuradas para la comprensión de textos.

Resulta muy importante que cada escolar establezca frente al texto, una meta, seleccione la rutina de pasos estructurados que aprendió en las clases anteriores, modifique en forma consciente la rutina en caso necesario y evalúe su proceso de comprensión.

Es necesario que el maestro o maestra en conjunto con sus estudiantes cree una agenda pictográfica para que escolares con TEA incorporen a sus modos de actuación el procedimiento necesario para abordar las tareas de comprensión textual en los contextos sociales. Para ello se proponen los siguientes procedimientos que constituirán rutinas aprendidas por escolares con TEA para enfrentarse a un texto en contextos sociales:

1. "¿Qué problema tengo?"

2. "Pienso cómo lo soluciono".

3. "Me apoyo en los recursos que tengo, en mis compañeros o compañeras, en otras personas adultas".

4. "Voy a intentarlo".

A fin de facilitar su enseñanza, recomendamos lo que señalan un grupo de personas autoras mexicanas al respecto:

- Motivación: indique la utilidad.

- Muestre y modele cada paso.

- Práctica guiada.

- Práctica independiente.

- Utilización en el día a día del aula [y en otros contextos]. (Escribano Burgos et ál., 2010, p. 167)

Quinta idea: El proceso de comprensión textual en escolares del primer ciclo con TEA ha de desarrollarse desde formas de organización que promuevan la interrelación de estudianteestudiante, docente-estudiante, estudiante-grupo, como componentes personales del proceso didáctico en función de la preparación para la vida social. 


\section{Argumentos}

Constituye una idea central porque atraviesa todo el proceso, por lo que el personal docente deberá, a partir de los objetivos y contenidos del grado y del sistema de clases propuesto, utilizar en cada clase todas las posibilidades que brinda el contenido para que escolares con TEA comprendan el texto en situaciones de la vida social.

La comprensión de las posibilidades de desarrollo de escolares con TEA, lo que esto significaría para la sociedad, lo que representa para sus familias y para ellos o ellas como seres humanos, si satisface las necesidades sociales e individuales en una plena integración social y la autonomía personal acorde con sus posibilidades.

Importante resulta la utilización de variadas formas de organización que promuevan la interrelación de estudiante, docente y grupo para desarrollar las relaciones sociales y la comunicación en escolares con TEA.

Es necesario que se relacionen los diferentes tipos de textos desde la clase de Lengua Española con las necesidades y situaciones de la vida social, se precisa la utilización de recursos didácticos y apoyos necesarios para que se empleen en la vida cotidiana, de manera que puedan aplicar rutinas estructuradas para la comprensión textual. No deben descuidarse en estos grupos escolares aspectos de la comunicación no verbal como la gestualidad y la comprensión de las señales del tránsito, por solo citar dos ejemplos.

Se recomienda el trabajo con los textos icónicos, avisos, propagandas, noticias que son tipologías a las cuales tiene acceso diariamente en su interacción social independiente.

Es necesario conseguir que las personas claves en su educación, como familiares y docentes aprovechen sistemáticamente todas las oportunidades para el desarrollo de la comprensión textual, a fin de conseguir el logro propuesto. Se debe estimular la comprensión de diversas tipologías textuales en los contextos naturales que favorecen la generalización de las agendas pictográficas de comprensión adquiridas en entornos más estructurados. Esta es la mejor manera de conseguir que aprendan nuevas competencias sociales, comunicativas y adaptativas.

Además, importante también resultará el desarrollo de las habilidades sencillas básicas para la realización de juicios, opiniones, valoraciones en las que escolares con TEA participen como individuos útiles dentro del grupo y la sociedad en la construcción de su propio aprendizaje y en el de las demás personas.

\section{Conclusiones}

Sin duda, el proceso de enseñanza- aprendizaje de la comprensión textual por escolares del primer ciclo con TEA potencia el desarrollo su integral, puesto que en la medida que recorra los niveles de este, desarrollará la comunicación y los comportamientos adecuados para la interacción en la vida social. 
http://doi.org/10.15359/ree.24-2.22

ROR: https://ror.org/01t466c14 Universidad Nacional, Costa Rica

http://www.una.ac.cr/educare

educare@una.cr

Desde estas ideas se pretendió esbozar una contextualización de las categorías didácticas del proceso de comprensión textual que perfile una valoración desarrolladora de escolares con TEA en su interacción social con otros sujetos (escolares - grupo - docente - familia - miembros de la comunidad) y su comportamiento en diferentes contextos (escuela - hogar - comunidad), lo que da lugar a que se expliciten los argumentos a tener en cuenta por unos sujetos y otros en el referido proceso.

Las ideas que se presentan generalizan y jerarquizan la concepción didáctica propuesta, las cuales se relacionan y se materializan en los argumentos, los cuales ofrecen una vía metodológica al personal docente para enseñar comprensión de textos a escolares con TEA en diferentes contextos.

Se distinguen por un enfoque didáctico, al ofrecer la utilización de variantes en los métodos y medios de enseñanza que promueven la utilización de pictogramas, agendas, calendarios y las formas de organización que estimulan la realización de actividades en grupo que favorece el desarrollo de la comunicación y socialización de escolares con TEA.

Además, posee un componente metodológico al brindar el tratamiento a través de un sistema de clases flexibles, que permiten la adaptación a las necesidades de escolares en todo el proceso de enseñanza-aprendizaje; tiene en cuenta las potencialidades, oportunidades y los conocimientos anteriores; promueve la actividad cognitiva; valora a escolares según sus capacidades, y esfuerzos y establece un ambiente favorable de relaciones que promueve la socialización, la comunicación y los comportamientos adecuados.

Escolares con TEA poseen características especiales en su desarrollo, como alteraciones en la socialización y comunicación, las cuales provocan limitaciones para el aprendizaje en general, y para la comprensión de diferentes tipos de textos. Por ello se recomienda usar, por ejemplo, textos en lenguaje facilitado, que permiten la comprensión de diferentes ejes temáticos relacionados con animales, medios de transporte, alimentos, etc., de manera que aprendan la comprensión de estos ejes hasta realizar generalizaciones; también se recomienda el trabajo con textos como cartas, invitaciones, despedidas, recetas, adivinanzas, anuncios, que constituyen textos importantes para su preparación para la vida social. Se potencian determinados elementos propiciadores. De ello depende, en gran medida, la calidad en las adquisiciones de esos contenidos, muchos de los cuales les facilitarán desenvolverse en todos los contextos. El grupo, mediante las interrelaciones que se establecen, impulsa el resultado de los apoyos, recursos y procederes pedagógicos.

El maestro o maestra que dirige el proceso de comprensión textual tiene como funciones esenciales mediar, diseñar, ofrecer ayudas pedagógicas y recursos didácticos a sus escolares para contribuir a su desarrollo; además, tiene el papel de orientar a la familia y coordinar con las personas de la comunidad para que participen, en conjunto, en el proceso de enseñanzaaprendizaje para aportar sus experiencias y vivencias. 
La familia debe implicarse, pues, a partir de su rol socializador, debe convertirse en un instrumento básico para apoyar el proceso de enseñanza-aprendizaje; el personal docente debe aprovechar las potencialidades y necesidades en función del aprendizaje, considerar las posibilidades que tiene cada una de las familias y sus integrantes, la contribución que puede hacer sobre la base de sus experiencias para enriquecer el conocimiento de escolares con TEA.

La comunidad es un espacio importante para el desarrollo de escolares con TEA. Se pueden realizar acciones de cooperación con las distintas instituciones que se encuentran en ella, como por ejemplo visitas a centros recreativos, culturales y deportivos, a barrios; debates para la concientización de las personas de la comunidad sobre el trastorno de autismo, donde el grupo escolar con TEA sea un ente activo para así contribuir a su preparación para la inclusión social.

La comprensión textual incluye a todos los sujetos y contextos expuestos anteriormente, ahí radica la importancia de considerarlos en el proceso de enseñanza, de manera que el centro de todo esa influencia sean escolares con TEA.

\section{Referencias}

Escribano Burgos, L., González del Yerro Valdés, A., Ortiz García, M., Simón Rueda, C., Tarragona Roig, M.y Uribe Franco, E. (2010). Laprevención de conductas desafiantes en la escuela infantil. Un enfoque proactivo. Fundación Educación y Desarrollo. https://www.orientacionandujar. es/2014/10/26/la-prevencion-de-conductas-desafiantes-en-la-escuela-infantil/

Chávez Rodríguez, J. A. (2011). Acercamiento necesario a la pedagogía general. Editorial Pueblo y Educación.

Guerra, S., Borges, S., Orosco, M., López, R. y Gayle, A. (2014). Actualidad de la atención educativa a los niños y adolescentes con necesidades educativas especiales. Editorial Pueblo y Educación.

Hernández Pérez, C., Berges Díaz, M., Aranda Cintra, B., Gayle Morejón, A., Rivera González, H. L. y Gracia Guerra, M. T. (2010). Básico curricular: Psicopedagogía. Educación Especial. III trayecto de formción especializada [Archivo en un blog]. http://ms-ubv-pnfe-girardot-edoaragua. blogspot.com/2010/12/basico-curricular-psicopedagogia.html

Labarrere Reyes, G. y Valdivia Pairol, G. E. (1988). Pedagogía. Editorial Pueblo y Educación.

Manzano Díaz, M. (2007). Estilos de aprendizaje, estrategias de lectura y su relación con el redimiento académico en la segunda lengua (Tesis doctoral). Universidad de Ciego de Ávila, Cuba. http://digibug.ugr.es/bitstream/handle/10481/1494/1665366x.pdf?sequence=1

Paz Aguilera, A. (2011). La filosofía de la educación en la escuela. Cuadernos de Educación y Desarrollo, 3(25). http://www.eumed.net/rev/ced/25/apa.htm 
http://doi.org/10.15359/ree.24-2.22

ROR: https://ror.org/01t466c14 Universidad Nacional, Costa Rica

http://www.una.ac.cr/educare

educare@una.cr

Rivière, A. (1997). Desarrollo normal y autismo (1/2). Definición, etiología, educación, familia, papel psicopedagógico en el autismo. Universidad Autónoma de Madrid.

Roméu Escobar, A. (Comp.). (2006). El enfoque cognitivo, comunicativo y sociocultural en la enseñanza de la lengua y literatura. Editorial Pueblo y Educación.

Van Dijk, T. A.(2000). El estudio del discurso. En T. A. van Dijk (Coord.), El discurso como estructura y proceso. Estudios sobre el discurso I. Una introducción multidisciplinaria (pp. 21-66). Gedisa.

Vigotski, L. S. (1987). Historia del desarrollo de las funciones psíquicas superiores. Científico Técnica. 